\title{
Nuclear Decay Data for the International Reactor Dosimetry Library for Fission and Fusion (IRDFF): Updated Evaluations of the Half-Lives and Gamma Ray Intensities
}

\author{
Valery P. Checheva and Nikolay K. Kuzmenko \\ Khlopin Radium Institute, 194021 St. Petersburg, Russia
}

\begin{abstract}
Updated evaluations of the half-lives and prominent gamma ray intensities have been presented for 20 radionuclides - dosimetry reaction residuals. The new values of these decay characteristics recommended for the IRDFF library were obtained using the approaches and methodology adopted by the working group of the Decay Data Evaluation Project (DDEP) cooperation. The experimental data published up to 2014 were taken into account in updated evaluations. The list of radionuclides includes ${ }^{3} \mathrm{H},{ }^{18} \mathrm{~F},{ }^{22} \mathrm{Na},{ }^{24} \mathrm{Na},{ }^{46} \mathrm{Sc}$, ${ }^{51} \mathrm{Cr},{ }^{54} \mathrm{Mn},{ }^{59} \mathrm{Fe},{ }^{57} \mathrm{Co},{ }^{60} \mathrm{Co},{ }^{57} \mathrm{Ni},{ }^{64} \mathrm{Cu},{ }^{88} \mathrm{Y},{ }^{132} \mathrm{Te},,{ }^{131} \mathrm{I},{ }^{140} \mathrm{Ba},{ }^{140} \mathrm{La},{ }^{141} \mathrm{Ce},{ }^{182} \mathrm{Ta},{ }^{198} \mathrm{Au}$.
\end{abstract}

\section{Introduction}

Recently the Nuclear Data Section of IAEA initiated a Coordinated Research Project (CRP) with the main goal to test, validate and improve the International Reactor Dosimetry Library for Fission and Fusion (IRDFF). Along with improving dosimetry reaction cross-sections, one of the objectives of the CRP is improving the Decay sub-library for IRDFF. Latter includes the 82 isotopes/isomers - dosimetry reaction residuals and additionally several new isotopes and isomers recommended for inclusion in IRDFF by 1st RCM (July 2013) [1].

Based on a comparison of the available ENSDF and DDEP evaluated data and analysis of the new published experimental information, we have determined the first group of 20 radionuclides to update their decay characteristics placed in the IRDFF Decay sub-library. The half-lives and the absolute intensity of the gamma rays (per decay) may be regarded as the main decay data which are required for this library. Therefore, updated evaluations were obtained for these characteristics in the current work based on experimental data and other information (compilations, analyses, corrections) published for chosen radionuclides up to 2014.

\section{Evaluation Techniques}

The authors used the approaches, programs and procedures adopted by the working group of the Decay Data Evaluation Project (DDEP) cooperation [2, 3].

For half-life evaluations based directly on published experimental data, the LWEIGHT computer

\footnotetext{
${ }^{a}$ Corresponding author: chechev@khlopin.ru
}

This is an Open Access article distributed under the terms of the Creative Commons Attribution License 2.0, which permits unrestricted use, distribution, and reproduction in any medium, provided the original work is properly cited. 
program [4], which uses the Limitation of Relative Statistical Weight method (LWM), was applied to average numbers throughout the evaluation. The uncertainty assigned to the average value is adopted greater than or equal to the smallest uncertainty of the values used to calculate the average, as the uncertainty of the best result ("smallest experimental uncertainty") reflects the type B standard uncertainty (systematic error of the method) and the recommended uncertainty cannot be less than it (for details of the evaluation methodology, see [4] and Sects. 2.1, 2.2 in [3]).

In relation to the problem discovered in the NIST calibration method [5], the 2014 corrected NIST half-life values [6] were introduced into the available experimental data sets, where required, and the re-evaluated half-life values have been obtained for long-lived radionuclides.

For absolute $\gamma$ ray intensity values, it is necessary to know not only the measured intensity values themselves but also the rest of the nuclide decay scheme. In some cases absolute $\gamma$ ray intensity values may be deduced directly from adopted decay scheme parameters, and in all cases the overall consistency of the decay scheme is the best check of the quality of $\gamma$ ray intensities [3]. The internal conversion coefficients (ICC) and their uncertainties used to obtain the absolute gamma ray intensities were interpolated from the tables of theoretical calculation results using the BrIcc computer program [7].

Below recommended values of the half-lives and prominent gamma ray intensities have been presented for each of the 20 nuclides with brief comments.

\section{Recommended Values of Half-lives and Prominent Gamma Ray Intensities}

\subsection{Radionuclide ${ }^{3} \mathrm{H}$}

Based on the DDEP ${ }^{3} \mathrm{H}$ decay data evaluation by V.P. Chechev [8], the ${ }^{3} \mathrm{H}$ half-life value recommended for use in the IRDFF Decay sub-library is 12.312 (25) years, or 4497 (9) days. No new measurements of the ${ }^{3} \mathrm{H}$ half-life have been published during the intervening period of 2006-2014.

\subsection{Radionuclide ${ }^{18} \mathrm{~F}$}

The DDEP ${ }^{18} \mathrm{~F}$ half-life evaluation by V. Chisté and M.M. Bé [9] was updated in this work taking into account the new measurements [10,11] additionally to the list given in [9]. The recommended value of ${ }^{18} \mathrm{~F}$ half-life has been obtained as the weighted average of 9 experimental values published since 1964 (the ratio of the reduced $\chi^{2} /\left(\chi^{2}\right)_{\text {crit }}$ is $2.4 / 2.5$ ), and it is 109.734 (14) min, or 1.82890 (23) hours.

The recommended value of the annihilation $511 \mathrm{keV}$ emission intensity in decay of ${ }^{18} \mathrm{~F}$ has been adopted from [3, 9]: $193.72(\mathbf{3 8}) \%$.

\subsection{Radionuclide ${ }^{22} \mathrm{Na}$}

The DDEP ${ }^{22} \mathrm{Na}$ half-life evaluation by M. Galán [12] was updated in this work using the 2014 corrected NIST half-life value of 2.6013 (19) years [6] (instead of 2.6037 (3) years previously reported). No new measurements have been published during the intervening period of 2009-2014. The recommended ${ }^{22} \mathrm{Na}$ half-life value of $\mathbf{2 . 6 0 2 0}$ (4) years, or 950.36 (15) days has been obtained as the weighted average of 9 available experimental values (the ratio of the reduced $\chi^{2} /\left(\chi^{2}\right)_{\text {crit }}$ is $0.5 / 2.5$ ).

The recommended values of the annihilation $511 \mathrm{keV}$ emission intensity of $\mathbf{1 8 0 . 7}$ (2)\% and $1274.54 \mathrm{keV}$ gamma ray emission probability of $99.94(\mathbf{1 3}) \%$ in decay of ${ }^{22} \mathrm{Na}$ have been adopted from $[3,12]$. No new measurements of the $\gamma$ ray intensities have been published during the intervening period of 2010-2014. 


\section{$15^{\text {th }}$ ISRD}

Table 1. Evaluated most intense $\gamma$ ray emission probabilities $(\mathrm{P} \gamma)$ in decay of ${ }^{24} \mathrm{Na}$.

\begin{tabular}{|c|c|}
\hline Energy, keV & $\mathbf{P} \gamma, \boldsymbol{\%}$ \\
\hline$\gamma \pm 511$ & $0.144(2)$ \\
\hline $1368.630(5)$ & $99.9934(5)$ \\
\hline $2754.049(13)$ & $99.863(3)$ \\
\hline $3866.12(4)$ & $0.066(2)$ \\
\hline
\end{tabular}

\subsection{Radionuclide ${ }^{24} \mathrm{Na}$}

The DDEP ${ }^{24} \mathrm{Na}$ half-life evaluation by R.G. Helmer and E. Schönfeld [9] was updated using the 2014 corrected NIST half-life value of 14.955 (7) hours [6] (instead of 14.9512 (32) hours previously reported) and taking into account one additional measurement by Ge detector in 2005 [13]. The recommended ${ }^{24} \mathrm{Na}$ half-life value of $\mathbf{1 4 . 9 5 8 1}(\mathbf{2 0})$ hours has been obtained as the weighted average (LWM) of 18 experimental values included (the ratio of the reduced $\chi^{2} /\left(\chi^{2}\right)_{\text {crit }}$ is $2.6 / 2.0$ ). It can be compared with earlier evaluations of 14.9574(20) h [9] and 14.997(12) h [14].

The recommended values of the gamma ray emission probabilities $(\mathrm{P} \gamma)$ in decay of ${ }^{24} \mathrm{Na}$ (Table 1) have been obtained from the analysis of the ${ }^{24} \mathrm{Na}$ decay scheme $[9,14]$ based on measured relative $\gamma$ ray intensities including the 2003 measurement [15], ICC interpolated from [7] and data on internal-pair formation ( $\alpha_{\pi}$ coefficients) [7,9]. " $\gamma \pm 511$ " denotes a photon emission produced by the annihilation of positrons (two $\gamma$ photons of energy $511 \mathrm{keV}$, see section 2.4 in [3] for details).

\subsection{Radionuclide ${ }^{46} \mathrm{Sc}$}

The DDEP ${ }^{46} \mathrm{Sc}$ half-life evaluation by R.G. Helmer [9] was updated using the 2014 corrected NIST half-life value of 83.84 (8) days [6] (instead of 83.83 (7) days previously reported). No new measurements have been published during the intervening period of 2004-2014. The recommended ${ }^{46} \mathrm{Sc}$ half-life value of $\mathbf{8 3 . 7 8 7}$ (16) days has been obtained as the weighted average (LWM) of 6 available experimental values since 1972 (the ratio of the reduced $\chi^{2} /\left(\chi^{2}\right)_{\text {crit }}$ is $2.5 / 3.0$ ).

The recommended values of the gamma ray emission probabilities $(\mathrm{P} \gamma)$ in decay of ${ }^{46} \mathrm{Sc}$ have been obtained from the intensity balance at each ${ }^{46} \mathrm{Ti}$ level [9] using ICC interpolated from [7]: $\mathrm{P} \gamma(889.27 \mathrm{keV})=\mathbf{9 9 . 9 8 3 7 4}(\mathbf{2 5}) \%, \mathrm{P} \gamma(1120.54 \mathrm{keV})=\mathbf{9 9 . 9 7}(\mathbf{2}) \%$.

\subsection{Radionuclide ${ }^{51} \mathrm{Cr}$}

The DDEP ${ }^{51} \mathrm{Cr}$ half-life evaluation by E. Schönfeld and R.G. Helmer [9] was updated using the 2014 corrected NIST half-life value of 27.70 (3) days [6] (instead of 27.7010 (12) days previously reported). No new measurements have been published during the intervening period of 2004-2014. The recommended ${ }^{51} \mathrm{Cr}$ half-life value of $\mathbf{2 7 . 7 0 6}$ (5) days has been obtained as the weighted average (LWM) of 21 available experimental values (the ratio of the reduced $\chi^{2} /\left(\chi^{2}\right)_{\text {crit }}$ is $4.9 / 1.9$ ).

The recommended value of $\mathrm{P} \gamma(320.08 \mathrm{keV})=\mathbf{9 . 8 9}(\mathbf{2}) \%$ has been obtained as the weighted average (LWM) of 10 available experimental values including the recent measurements $[16,17]$ (the ratio of the reduced $\chi^{2} /\left(\chi^{2}\right)_{\text {crit }}$ is $\left.0.5 / 2.4\right)$.

\subsection{Radionuclide ${ }^{54} \mathrm{Mn}$}

The DDEP ${ }^{54} \mathrm{Mn}$ half-life evaluation by R.G. Helmer and E. Schönfeld [9] was updated using the 2014 corrected NIST half-life value of 311.94(17) days [6] and taking into account the recent measurements 
since 2000 [18-20]. The recommended ${ }^{54} \mathrm{Mn}$ half-life value of 312.19 (3) days has been obtained as the weighted average (LWM) of 14 available experimental values including [18-20] (the ratio of the reduced $\chi^{2} /\left(\chi^{2}\right)_{\text {crit }}$ is $\left.0.7 / 2.0\right)$.

The $834.85 \mathrm{keV} \gamma$ ray emission probability of 99.9752 (5)\% has been deduced from the adopted electron capture transition probability to the ${ }^{54} \mathrm{Cr} 834.85 \mathrm{keV}$ level (99.9997 (3)\%) and the total ICC $\alpha_{T}$ interpolated from [7].

\subsection{Radionuclide ${ }^{59} \mathrm{Fe}$}

The DDEP ${ }^{59} \mathrm{Fe}$ half-life evaluation by M.M. Bé and V. Chisté [9] was updated using the 2014 corrected NIST half-life value of 44.508 (16) days [6] (instead of 44.5074 (72) days previously reported). No new half-life measurements have been published during the intervening period of 2004-2014. The recommended ${ }^{59} \mathrm{Fe}$ half-life value of $\mathbf{4 4 . 4 9 4}$ (12) days has been obtained as the weighted average (with the external uncertainty) of the set of 11 experimental values (the ratio of the reduced $\chi^{2} /\left(\chi^{2}\right)_{\text {crit }}$ is 6.2/2.3).

The evaluated gamma ray emission probabilities $(\mathrm{P} \gamma)$ in decay of ${ }^{59} \mathrm{Fe}$ [9] were obtained in this work using averaged measured relative gamma ray intensities and the ICC values interpolated from [7]: $\mathrm{P} \gamma(1099.24 \mathrm{keV})=\mathbf{5 6 . 5 1}(\mathbf{3 1}) \%, \mathrm{P} \gamma(1291.59 \mathrm{keV})=\mathbf{4 3 . 2 3}(\mathbf{3 3}) \%$. The uncertainties are the smallest uncertainties of the experimental values used to calculate the average. No new measurements have been published during the intervening period of 2004-2014.

\subsection{Radionuclide ${ }^{57}$ Co}

The DDEP ${ }^{57}$ Co half-life evaluation by V.P. Chechev and N.K. Kuzmenko [9] was updated using the 2014 corrected NIST half-life value of 271.87 (44) days [6] (instead of 272.11 (26) days previously reported) and taking into account one additional measurement published in 2012 [21]. The recommended ${ }^{57}$ Co half-life value of $\mathbf{2 7 1 . 8 0}$ (4) days has been obtained as the weighted average (LWM) of 7 experimental values including [21] (the ratio of the reduced $\chi^{2} /\left(\chi^{2}\right)_{\text {crit }}$ is $1.0 / 2.8$ ). The uncertainty of the recommended value is the smallest uncertainty of the experimental values used.

The evaluated gamma ray emission probabilities $(\mathrm{P} \gamma)$ in decay of ${ }^{57} \mathrm{Co}$ [9] were corrected in this work to the ICC values interpolated from [7]: $\mathrm{P} \gamma(14.413 \mathrm{keV})=\mathbf{9 . 1 7}(\mathbf{1 3}) \%, \mathrm{P} \gamma(122.06 \mathrm{keV})=$ $\mathbf{8 5 . 5 1}(\mathbf{6}) \%, \mathrm{P} \gamma(136.47 \mathrm{keV})=\mathbf{1 0 . 7 1}(\mathbf{1 5}) \%$.

\subsection{Radionuclide ${ }^{60} \mathrm{Co}$}

The DDEP ${ }^{60}$ Co half-life evaluation by R.G. Helmer [8] was updated using the 2014 corrected NIST half-life value of 1923.8 (12) days [6] (instead of 1925.20 (25) days previously reported). No new half-life measurements have been published during the intervening period of 2006-2014. The recommended ${ }^{60} \mathrm{Co}$ half-life value of $\mathbf{1 9 2 5 . 2}$ (3) days, or $\mathbf{5 . 2 7 1 0}$ (8) years has been obtained as the weighted average (LWM) of 8 experimental values (the ratio of the reduced $\chi^{2} /\left(\chi^{2}\right)_{\text {crit }}$ is $1.5 / 2.6$ ). The uncertainty of the recommended value is the smallest uncertainty of the experimental values used.

The evaluated gamma ray emission probabilities $(\mathrm{P} \gamma)$ in decay of ${ }^{60} \mathrm{Co}$ [8] were corrected in this work to the ICC values interpolated from [7]: $\mathrm{P} \gamma(1173.23 \mathrm{keV})=\mathbf{9 9 . 8 5}(3) \%, \mathrm{P} \gamma(1332.49 \mathrm{keV})=$ $99.9826(2) \%$. 


\section{$15^{\text {th }}$ ISRD}

Table 2. Evaluated most intense $\gamma$ ray emission probabilities $(\mathrm{P} \gamma)$ in decay of ${ }^{88} \mathrm{Y}$.

\begin{tabular}{|c|c|}
\hline Energy, keV & $\mathbf{P} \boldsymbol{\gamma}, \boldsymbol{\%}$ \\
\hline$\gamma \pm 511$ & $0.456(28)$ \\
\hline $898.042(11)$ & $93.89(30)$ \\
\hline $1836.070(8)$ & $99.346(25)$ \\
\hline $2734.092(8)$ & $0.608(25)$ \\
\hline
\end{tabular}

\subsection{Radionuclide ${ }^{57} \mathrm{Ni}$}

As no new ${ }^{57} \mathrm{Ni}$ half-life and gamma ray intensities' measurements have been published during the intervening period of 2004-2014, the values of these decay characteristics recommended for IRDFF library have been adopted from the DDEP evaluation by Shiu-Chin Wu presented in [9] on pages 91-98: $\mathrm{T}_{1 / 2}\left({ }^{57} \mathrm{Ni}\right)=35.9(3)$ hours, $\mathrm{P} \gamma(127.16 \mathrm{keV})=\mathbf{1 6 . 0}(\mathbf{5}) \%, \mathrm{P} \gamma \pm(511 \mathrm{keV})=86.8$ (12)\%, $\mathrm{P} \gamma(1377.6 \mathrm{keV})=\mathbf{8 1 . 2}(\mathbf{6}) \%, \mathrm{P} \gamma(1757.6 \mathrm{keV})=\mathbf{6 . 1}(\mathbf{4}) \%, \mathrm{P} \gamma(1919.6 \mathrm{keV})=\mathbf{1 2 . 5}(\mathbf{5}) \%$.

\subsection{Radionuclide ${ }^{64} \mathrm{Cu}$}

The DDEP ${ }^{64} \mathrm{Cu}$ half-life evaluation by M.M. Bé and R.G. Helmer presented in [22] on pages 1319 was updated taking into account one new measurement [23] additionally to the list given in [22]. The recommended ${ }^{64} \mathrm{Cu}$ half-life value of $\mathbf{1 2 . 7 0 0}$ (2) hours has been obtained as the weighted average (LWM) of 9 available experimental values since 1972 (the ratio of the reduced $\chi^{2} /\left(\chi^{2}\right)_{\text {crit }}$ is $0.2 / 2.5$ ). The uncertainty of the recommended value is the smallest uncertainty of the experimental values used.

The recommended values of the annihilation $511 \mathrm{keV}$ emission intensity of 35.04 (30)\% and $1345.8 \mathrm{keV}$ gamma ray emission probability of $\mathbf{0 . 4 7 4 8}\left(\mathbf{3 4 )} \%\right.$ in decay of ${ }^{64} \mathrm{Cu}$ have been adopted from $[3,22]$. No new measurements of the $\gamma$ ray intensities have been published during the intervening period of 2010-2014.

\subsection{Radionuclide ${ }^{88} Y$}

The first DDEP evaluation of ${ }^{88} \mathrm{Y}$ decay data was done by E. Schönfeld in 1998 with minor update in 2004 [9]. The current evaluation has been completed in March 2014 with a literature cut-off by the same date.

The recommended ${ }^{88} \mathrm{Y}$ half-life value of $\mathbf{1 0 6 . 6 3}$ (5) days has been obtained as the weighted average (LWM) of 7 experimental values including the recent ones $[6,24]$ (the ratio of the reduced $\chi^{2} /\left(\chi^{2}\right)_{\text {crit }}$ is $0.2 / 2.8$ ). The uncertainty of the recommended value is the smallest uncertainty of the experimental values used.

The recommended values of the gamma ray emission probabilities $(\mathrm{P} \gamma)$ in decay of ${ }^{88} \mathrm{Y}$ (Table 2) have been obtained using averaged measured relative $\gamma$ ray intensities and ICC and $\alpha_{\pi}$ coefficients interpolated from [7].

\subsection{Radionuclide ${ }^{132} \mathrm{Te}$}

As no new ${ }^{132} \mathrm{Te}$ half-life and gamma ray intensities measurements have been published during the intervening period of 2009-2014, the values of these decay characteristics recommended for IRDFF library have been adopted from the DDEP evaluation by A.L. Nichols [22]: $\mathrm{T}_{1 / 2}$ $\left({ }^{132} \mathrm{Te}\right)=3.230$ (13) days, $\mathrm{P} \gamma(49.72 \mathrm{keV})=\mathbf{1 5 . 1}(\mathbf{3}) \%, \mathrm{P} \gamma(111.8 \mathrm{keV})=\mathbf{1 . 8 5}$ (18)\%, $\mathrm{P} \gamma(116.3 \mathrm{keV})=\mathbf{1 . 9 7}(\mathbf{7}) \%, \mathrm{P} \gamma(228.33 \mathrm{keV})=\mathbf{8 8 . 1 2}(\mathbf{1 3}) \%$. 
Table 3. Evaluated most intense $\gamma$ ray emission probabilities $(\mathrm{P} \gamma)$ in decay of ${ }^{140} \mathrm{Ba}$.

\begin{tabular}{|c|c|}
\hline Energy, keV & $\mathbf{P} \boldsymbol{\gamma}, \boldsymbol{\%}$ \\
\hline $29.9641(6)$ & $14.5(4)$ \\
\hline $162.6591(19)$ & $6.32(14)$ \\
\hline $304.971(30)$ & $4.34(9)$ \\
\hline $423.79(4)$ & $3.14(6)$ \\
\hline $437.666(30)$ & $1.95(4)$ \\
\hline $537.261(25)$ & $24.6(5)$ \\
\hline
\end{tabular}

\subsection{Radionuclide ${ }^{131}$ I}

A very recent DDEP evaluation of ${ }^{131}$ I decay data by V. Chisté and M.M. Bé has been placed on the DDEP web site (http://www.nucleide.org/DDEP_WG/DDEPdata.htm). The evaluated ${ }^{131}$ I half-life and prominent gamma ray intensities are recommended for IRDFF library from this evaluation: $\mathrm{T}_{1 / 2}\left({ }^{131} \mathrm{I}\right)=\mathbf{8 . 0 2 3 3}(\mathbf{1 9})$ days, $\mathrm{P} \gamma(80.19 \mathrm{keV})=\mathbf{2 . 6 0 7}(\mathbf{3 5}) \%, \mathrm{P} \gamma(284.3 \mathrm{keV})=\mathbf{6 . 1 4}$ (6) \%, $\mathrm{P} \gamma(364.5 \mathrm{keV})=\mathbf{8 1 . 2}(\mathbf{5}) \%, \mathrm{P} \gamma(637.0 \mathrm{keV})=\mathbf{7 . 1 2}(\mathbf{7}) \%, \mathrm{P} \gamma(722.9 \mathrm{keV})=\mathbf{1 . 7 8 6}(\mathbf{1 9}) \%$.

\subsection{Radionuclide ${ }^{140} \mathrm{Ba}$}

The initial DDEP evaluation of ${ }^{140} \mathrm{Ba}$ decay data was done by R.G. Helmer in 2003 [9]. The current evaluation has been completed by the authors in March 2014 with a literature cut-off by the same date.

The recommended ${ }^{140} \mathrm{Ba}$ half-life value of $\mathbf{1 2 . 7 5 3}$ (5) days has been obtained as the weighted average (LWM) of 5 experimental values including the 2014 corrected NIST half-life value of 12.753(12) days [6] (instead of 12.7527(23) days previously reported). The ratio of the reduced $\chi^{2} /\left(\chi^{2}\right)_{\text {crit }}$ is $1.2 / 3.3$.

The recommended values of the gamma ray emission probabilities $(\mathrm{P} \gamma)$ in decay of ${ }^{140} \mathrm{Ba}$ (Table 3) have been obtained using averaged measured relative $\gamma$ ray intensities and ICC interpolated from [7]. The relative emission probabilities (relative to the $537.3 \mathrm{keV} \gamma$ ray intensity taken as 100 ) have been scaled by 0.246 (5) \% . This normalization factor was obtained from the transition intensity balance to the ground state of ${ }^{140} \mathrm{La}$.

\subsection{Radionuclide ${ }^{140}$ La}

The initial DDEP evaluation of ${ }^{140}$ La decay data was done by R.G. Helmer in 2003 [9]. The current evaluation has been completed by the authors in April 2014 with a literature cut-off by the same date. The evaluators have accepted fully the Helmer's approach to the evaluation of gamma ray energies and emission probabilities and associated values updating only the evaluated values of ICC and other decay characteristics due to new publications of 2004-2014.

The recommended ${ }^{140}$ La half-life value of $\mathbf{4 0 . 2 8 6}(5)$ hours, or 1.67858 (21) days, has been obtained as the weighted average (LWM) of 13 experimental values including the 2014 corrected NIST halflife value of 40.294(7) days [6] (instead of 40.293(12) days previously reported). The ratio of the reduced $\chi^{2} /\left(\chi^{2}\right)_{\text {crit }}$ is $1.7 / 2.1$. The uncertainty of the recommended value is the smallest uncertainty of the experimental values used.

The recommended values of the most intense gamma ray emission probabilities $(\mathrm{P} \gamma)$ in decay of ${ }^{140} \mathrm{La}$ are as follows: $\mathrm{P} \gamma(328.76 \mathrm{keV})=\mathbf{2 0 . 8}(\mathbf{3}) \%, \mathrm{P} \gamma(487.02 \mathrm{keV})=4 \mathbf{6 . 1}(\mathbf{5}) \%, \mathrm{P} \gamma(815.78 \mathrm{keV})=$ $23.72(\mathbf{2 0}) \%, \mathrm{P} \gamma(1596.2 \mathrm{keV})=\mathbf{9 5 . 4 2 8}(\mathbf{2 5}) \%$. 


\section{$15^{\text {th }}$ ISRD}

Table 4. Recommended $\gamma$ ray emission probabilities $(\mathrm{P} \gamma)$ in decay of ${ }^{198} \mathrm{Au}$.

\begin{tabular}{|l|l|}
\hline Energy, keV & $\mathbf{P} \gamma, \%$ \\
\hline $411.80205(17)$ & $95.62(6)$ \\
\hline $675.8836(7)$ & $0.804(5)$ \\
\hline $1087.6842(7)$ & $0.1591(21)$ \\
\hline
\end{tabular}

\subsection{Radionuclide ${ }^{141} \mathrm{Ce}$}

The initial DDEP evaluation of ${ }^{141}$ Ce decay data by E. Schönfeld [25] was updated by V.P. Chechev in 2013 [26]. The ${ }^{141} \mathrm{Ce}$ half-life value of $\mathbf{3 2 . 5 0 4}$ (13) days recommended in this work for the IRDFF library has been obtained as the weighted average (LWM) of 8 experimental values including the new ones $[6,27]$ (the ratio of the reduced $\chi^{2} /\left(\chi^{2}\right)_{\text {crit }}$ is $\left.0.25 / 2.6\right)$. The recommended value of the gamma ray emission probability $\mathrm{P} \gamma(145.44 \mathrm{keV})$ of $\mathbf{4 8 . 2 9}(\mathbf{3 0}) \%$ has been obtained as the weighted average of 4 published experimental values [25] (the ratio of the reduced $\chi^{2} /\left(\chi^{2}\right)_{\text {crit }}$ is $0.28 / 3.8$ ). The uncertainties of both recommended values are the smallest uncertainties of the experimental values used.

\subsection{Radionuclide ${ }^{182} \mathrm{Ta}$}

As no new ${ }^{182} \mathrm{Ta}$ half-life and gamma ray intensities' measurements have been published during the intervening period of 2011-2014, the values of these decay characteristics recommended for IRDFF library have been adopted from the DDEP evaluation by V. Chisté and M.M. Bé [22]: $\mathrm{T}_{1 / 2}\left({ }^{182} \mathrm{Ta}\right)=\mathbf{1 1 4 . 6 1}(\mathbf{1 3})$ days, $\mathrm{P} \gamma(67.7497 \mathrm{keV})=\mathbf{4 3 . 6}(\mathbf{1 5}) \%, \mathrm{P} \gamma(100.106 \mathrm{keV})=\mathbf{1 4 . 2 2}$ (16)\%, P $\gamma(1121.29 \mathrm{keV})=35.17(33) \%, P \gamma(1189.04 \mathrm{keV})=\mathbf{1 6 . 5 8}(\mathbf{1 6}) \%, \mathrm{P} \gamma(1221.40 \mathrm{keV})=$ $27.27(27) \%, \mathrm{P} \gamma(1231.00 \mathrm{keV})=\mathbf{1 1 . 6 2}(\mathbf{1 1}) \%$.

\subsection{Radionuclide ${ }^{198} \mathrm{Au}$}

A very recent DDEP evaluation of ${ }^{198} \mathrm{Au}$ decay data by the authors of this work has been placed on the DDEP web site (http://www. nucleide.org/DDEP_WG/DDEPdata.htm). The evaluated ${ }^{198} \mathrm{Au}$ halflife of $\mathbf{2 . 6 9 4 3 ~ ( 3 ) ~ d a y s ~ a n d ~ g a m m a ~ r a y ~ e m i s s i o n ~ p r o b a b i l i t i e s ~ ( T a b l e ~ 4 ) ~ a r e ~ r e c o m m e n d e d ~ f o r ~ t h e ~ I R D F F ~}$ library from the above evaluation.

\section{Conclusion}

The evaluated half-lives and prominent gamma ray intensities for 20 radionuclides - dosimetry reaction residuals - are recommended for the IRDFF library. The computer programs, approaches, procedures and methodology adopted by the working group of the Decay Data Evaluation Project (DDEP) cooperation have been used in these evaluations based on the experimental data and other information published up to 2014 .

This work was funded through the IAEA Research Contract 17762, which is gratefully acknowledged.

\section{References}

[1] Summary Report of the First Research Coordination Meeting on Testing and Improving the International Reactor Dosimetry and Fusion File (IRDFF), IAEA NDC (NDS)-0639 (Prepared by A. Trkov, L.R. Greenwood, S.P. Simakov, IAEA Nuclear Data Section, 2013) 
[2] M.-M. Bé, R.G. Helmer, J. Nucl. Science Tech. Supp. 2, 481 (2002)

[3] M.-M. Bé, V.P. Chechev, Nucl. Instrum. Methods Phys. Res. A728, 157 (2013)

[4] E. Browne, Limitation of Relative Statistical Weights, a method for evaluating discrepant data, INDC(NDS)-363 (IAEA, March 1988). T.D. MacMahon and E. Browne, LWEIGHT, a computer program to calculate averages, Version 1.3 (IAEA, March 2000)

[5] M.P. Unterweger, R. Fitzgerald, Appl. Radiat. Isot. 70, 1892 (2012)

[6] M.P. Unterweger, R. Fitzgerald, Appl. Radiat. Isot. 87, 92 (2014)

[7] T. Kibédi, T.W. Burrows, M.B. Trzhaskovskaya, P.M. Davidson, C.W. Nestor Jr. Nucl. Instrum. Methods Phys. Res. A589, 202 (2008)

[8] M.-M. Bé, V. Chisté, C. Dulieu, E. Browne, V. Chechev, N. Kuzmenko, R. Helmer, F. Kondev, D. MacMahon, K.B. Lee, Table of Radionuclides (Vol. $3-A=3$ to 244). (Monographie BIPM-5, Vol. 3, Sevres: Bureau International des Poids et Mesures, 2006)

[9] M.-M. Bé, V. Chisté, C. Dulieu, E. Browne, V. Chechev, N. Kuzmenko, R. Helmer, A. Nichols, E. Shönfeld, and R. Dersch, Table of Radionuclides (Vol. $1-A=7$ to 150). (Monographie BIPM-5, Vol. 1, Sevres: Bureau International des Poids et Mesures, 2004)

[10] E. Garcia-Torano, V. Peyres Medina, M. Roteta Ibarra, Appl. Radiat. Isot. 68, 1561 (2010)

[11] J. Han, K.B. Lee, T.S. Park, J.M. Lee, P.J. Oh, S.H. Lee, Y.S. Kang, J.K. Ahn, Appl. Radiat. Isot. 70, 2581 (2012)

[12] M.-M. Bé, V. Chisté, C. Dulieu, X. Mougeot, E. Browne, V. Chechev, N. Kuzmenko, F. Kondev, A. Luca, M. Galan, A. Arinc, and X. Huang, Table of Radionuclides (Vol. $5-A=22$ to 244). (Monographie BIPM-5, Vol. 5, Sevres: Bureau International des Poids et Mesures, 2010)

[13] R.M. Lindstrom, M. Blaauw, M.P. Unterweger, J. Radioanal. Nucl. Chem. 263, 311 (2005)

[14] R.B. Firestone, Nuclear Data Sheets 108, 2319 (2007)

[15] E.M. Epp, H.C. Griffin, Nucl. Instrum. Methods Phys. Res. A505, 9 (2003)

[16] P. Yalcin, Y. Kurucu, Appl. Radiat. Isot. 62, 63 (2005)

[17] D.S. Moreira, M.F. Koskinas, I.M. Yamazaki, M.S. Dias, Appl. Radiat. Isot. 68, 596 (2010)

[18] C.A. Huh, L.F. Liu, J. Radioanal. Nucl. Chem. 246, 229 (2000)

[19] M.A.L. da Silva, R. Poledna, A. Iwahara, C.J. da Silva, J.U. Delgado, R.T. Lopes. Appl. Radiat. Isot. 64, 1440 (2006)

[20] R. Van Ammel, J. Paepen, S. Pommé, G. Sibbens, Appl. Radiat. Isot. 68, 2387 (2010)

[21] C.J. da Silva, A. Iwahara, D.S. Moreira, J.U. Delgado, R.S. Gomes, Appl. Radiat. Isot. 70, 1924 (2012)

[22] M.-M. Bé, V. Chisté, C. Dulieu, X. Mougeot, V.P. Chechev, N.K. Kuzmenko, F.G. Kondev, A. Luca, M. Galan, A.L. Nichols, A. Arinc, A. Pearce, X. Huang, B. Wang, Table of Radionuclides (Vol. $6-A=22$ to 242). (Monographie BIPM-5, Vol. 6, Sevres: Bureau International des Poids et Mesures, 2011)

[23] M.N. Amiot, M.M. Bé, T. Branger, P. Cassette, M.C. Lepy, Y. Menesguen, I. Da Silva, Nucl. Instrum. Methods Phys. Res. A684, 97 (2012)

[24] M.N. Amiot, J. Bouchard, M.-M. Bé, J.B. Adamo, Appl. Rad. Isotop. 62, 11 (2005)

[25] M.-M. Bé, B. Duchemin, J. Lame, C. Morillon, F. Piton, E. Browne, V. Chechev, R. Helmer, E. Schönfeld, Table of Radionuclides, CEA-ISBN 2-7272-0200-8 (1999)

[26] M.-M. Bé, V. Chisté, C. Dulieu, X. Mougeot, V.P. Chechev, F.G. Kondev, A.L. Nichols, X. Huang, B. Wang, Table of Radionuclides (Vol. $7-A=14$ to 245). (Monographie BIPM-5, Vol. 7, Sevres: Bureau International des Poids et Mesures, 2013)

[27] S. Torrel, K.S. Krane, Phys. Rev. C 86, 034340 (2012) 